\title{
An Adaptive TDMA Slot Assignment Strategy in Vehicular Ad Hoc Networks
}

\author{
M. Hadded ${ }^{1}$, R. Zagrouba ${ }^{2}$, A. Laouiti ${ }^{3}$, P. Muhlethaler ${ }^{4}$, \\ and L. A. Saïdane ${ }^{5}$ \\ ${ }^{1}$ RAMSIS Team, CRISTAL Laboratory, 2010 Campus University, \\ Manouba, Tunisia \\ ${ }^{2}$ Higher Institute of Computer Science, \\ Ariana, Tunisia \\ ${ }^{3}$ TELECOM SudParis, CNRS Samovar, \\ UMR 5157, France \\ ${ }^{4}$ INRIA, BP 105. 78153 Le Chesnay Cedex, \\ Paris-Rocquencourt, France \\ ${ }^{5}$ National School of Computer Science, 2010 Campus University, \\ Manouba, Tunisia
}

Received 23 February 2014; Accepted 25 May 2014

Publication 4 August 2014

\begin{abstract}
Improving road safety is among the main objectives of Vehicular Ad-hoc NETworks (VANETs) design. This objective would be achieved essentially by the use of efficient safety applications which should be able to wirelessly broadcast warning messages between neighbouring vehicles in order to inform drivers about a dangerous situation in a timely manner. To insure their efficiency, safety applications require reliable periodic data dissemination with low latency. Medium Access Control (MAC) protocols play a primary role to provide efficient delivery and avoid as much as possible data packet loss. In fact, in distributed MAC approaches, packet loss is a consequence of collisions resulted from well known situations of the exposed and hidden node situations. This paper introduces an Adaptive TDMA Slot Assignment Strategy (ASAS) for VANET based on clustering of vehicles. The main aim of this work is to provide a MAC layer protocol that can reduce inter-cluster

Journal of Machine to Machine Communications, Vol. 1 , 175-194.

doi: 10.13052/jmmc2246-137X.126

(c) 2014 River Publishers. All rights reserved.
\end{abstract}


interference under different traffic loading conditions without having to use expensive spectrum and complex mechanisms such as CDMA or OFDMA. An analysis and simulation results are presented to evaluate the performance of ASAS. Moreover, we compare its performance with two TDMA MAC protocols DMMAC and VeMAC.

Keywords: VANET, QoS, MAC protocols, CDMA, TDMA, FDMA.

\section{Introduction}

The Vehicular Ad-hoc NETwork (VANET) is a sub class of Mobile Ad-hoc NETwork (MANET), which has some special characteristics such as the high dynamicity of the nodes, the lack of infrastructure and diverse quality of service (QoS) requirements. In VANETs, communications can either be between nearby vehicles V2V (Vehicle To Vehicle) or between vehicles and road side units V2I [9] (Vehicle To Infrastructure). Due to the importance of $\mathrm{V} 2 \mathrm{~V}$ communications, several research projects are underway to standardize V2V communication in Europe and around the world such as the Car2Car consortium [5] which seeks to improve road safety. In the US, the FCC (Federal Communication Commission) [2] established the DSRC service (Dedicated Short Range Communications) in 2003. The DSRC [3] radio technology is defined in the frequency band of $5.9 \mathrm{GHz}$ with a total bandwidth of $75 \mathrm{MHz}$. This band is divided into 7 channels of $10 \mathrm{MHz}$ for each one. These channels comprise one Control CHannel $(\mathrm{CCH})$ and six Service CHannels (SCHs), each one offering a throughput from 6 to $27 \mathrm{Mbps}$. The $\mathrm{CCH}$ is reserved for the network management messages, but is also used to transmit messages of high priority. The six SCHs are dedicated to data transmission.

However, the V2V communication is based on the exchange of beacon messages (current status, aggregate data, and emergency messages). If several vehicles broadcast these messages at the same time, then a collision will occur. Thus, it is crucial to avoid collision on the Control CHannel $\mathrm{CCH}$ in order to ensure a fast and reliable safety messages exchange. To provide a QoS and reduce the collision on the $\mathrm{CCH}$, we introduce an adaptive TDMA slot allocation strategy that takes into account the specificity of VANETs networks. The strategy proposed operates at the stable clusters heads which

are responsible for assigning disjoint sets of time slots to the members of their clusters according to their directions and positions. Thus, by using 
a centralized means of slots reservation, we ensure an efficient utilization of the time slots and thereby decrease the rate of merging collision [13] and hidden node collisions caused by vehicles moving in opposite directions.

The remainder of this paper is organized as follows. Section 2 reviews related works on MAC protocols in VANET. Section 3 sets out the challenges of TDMA based MAC solution deployment. We give a detailed description of ASAS in Section 4. In Section 5, we present and discuss the simulation results. Conclusion and perspectives are presented in Section 6.

\section{Related Work}

Several distributed MAC protocols have been designed for inter-vehicle communications. They can be classified into three categories, the contentionbased medium access method CSMA/CA such as IEEE 802.11p [6], and the contention-free medium access method using Time Division Multiple Access TDMA, such as VeMAC [13, 14], TC-MAC [15]. The third category is a hybrid of the two previous methods such as DMMAC [17].

Recently, the TGp Task Group of IEEE [10] has proposed the IEEE 802.11p [6] to support VANET communications. Based IEEE 802.11, this standard tends to improve the QoS by using different messages priorities. In fact, IEEE 802.11p implements the Enhanced Distributed Channel Access EDCA [7] technique for packet prioritization. Nevertheless, a major problem of the IEEE 802.11p standard comes from the lack of bounded channel access delays guarantees [1], since it is based on a contention MAC method.

In [13] the authors propose a contention-free medium access control protocol for VANET called VeMAC. Vehicles in VeMAC are equipped with two radio interfaces, where the first is always tuned to the control channel $\mathrm{CCH}$ while the second one can be tuned to any service channel. $\mathrm{CCH}$ slot allocation is performed in a distributed manner where each vehicle randomly gets an available time slot. It is not the case for the SCH slot allocation. This task is ensured by service providers in a centralized way. However, packets transmitted by VeMAC on the $\mathrm{CCH}$ are large (Vehicle ID, current position, set of one-hop neighbours and the time slot used by each node within onehop neighbourhood), which induces a high overhead on the $\mathrm{CCH}$. Moreover, its random slot allocation technique is not efficient due to the appearance of free slots.

Günter and al. [14] propose a cluster based medium access control protocol (CBMAC). In their protocol each cluster head is responsible for time slot 
assignment to its cluster members for messages transmission. The aim of this protocol is to limit the effect of the hidden node problem and offer a fair medium access. In CBMAC protocol, the access time is divided into periodic frames and each frame is divided into time slots. The $\mathrm{CH}$ generates and manages the TDMA slot reservation schedule for its vehicles members according to the amount of data needed to send.

Recently a novel multi-channel MAC protocol called TDMA clusterbased MAC (TC-MAC) for Vehicular Ad hoc NETworks has been proposed by Almalag and al. [15]. It is based on stable clusters heads which are in charge of TDMA time slot assignment. TC-MAC provides an efficient time slots utilization for the exact number of active vehicles. Unlike WAVE MAC architecture, in TC-MAC protocol, the frame is not divided into two intervals CCHI and SCHI. In fact, each vehicle may switch to the Control Channel $(\mathrm{CCH})$ or to a specific service channel $(\mathrm{SCH})$ when needed during the time slot.

In [17], the authors introduce the Dedicated Multi-channel MAC (DMMAC) protocol. The DMMAC architecture is similar to WAVE MAC with the difference that in DMMAC, the $\mathrm{CCH}$ Interval is divided into an Adaptive Broadcast Frame (ABF) and a Contention-based Reservation Period (CRP). The ABF period composed of time slots, each time slot is dynamically reserved by an active vehicle as its Basic Channel $(\mathrm{BCH})$ for collisionfree delivery of the safety message or other control messages. The CRP employs CSMA/CA to organize its channel access. During the CRP, the vehicles negotiate and reserve the network resources on SCHs for non-safety applications. In addition, it is restricted to the only scenario of a straight highway road with an available number of slots higher than the maximum number of cars.

\section{TDMA based MAC Protocol Challenges in VANETs Networks}

The first aim of a MAC protocol for VANET is to ensure that each vehicle is granted an access to the channel in a bounded delay in order to send safety messages without collisions. TDMA is a method that can be used to assign one time slot to each active vehicle. We will study below the challenges of MAC solutions in VANETs focusing particularly on the TDMA technique. In the following we highlight the major problems faced in the case of a distributed TDMA slot allocation technique and in the case of a cluster based TDMA slot allocation technique. 


\subsection{Distributed TDMA Slot Allocation Challenges}

When a distributed scheme is used to allocate a time slot, two types of collision on the time slots can happen [12] : access collision between vehicles trying to allocate the same available time slots, and merging collision between vehicles using the same time slots. The access collision problem [13] occurs when two or more vehicles within the same two-hop neighbourhood set attempt to allocate the same available time slot. This problem is likely to happen when a distributed scheme is used to allow the vehicle to reserve a time slot. As shown in Figure 1., the two vehicles attempt to access the same slot when they are within two-hops range. While the merging collision [12] is a basic problem for vehicular ad hoc network, this problem occurs when two vehicles in different two-hops sets using the same time slot become members of the same two-hop set due to their mobility. Figure 2 shown an example of the merging collision problem, when vehicle B in the first two-hop set is moving in opposite direction to vehicle $\mathrm{E}$ in the second two-hop set which is using the same time slot as B. Since B and E become members of the same two-hop set, then a collision will occur at vehicle D.

\subsection{Centralized TDMA Slot Allocation}

When the slot assignment schedule is centralized in the clusters heads, an inter-clusters interference problem can arise. There are two types of intercluster interference [4]: One Hop neighbouring Collision and Hidden Node Collision.



Figure 1 Access collision problem 


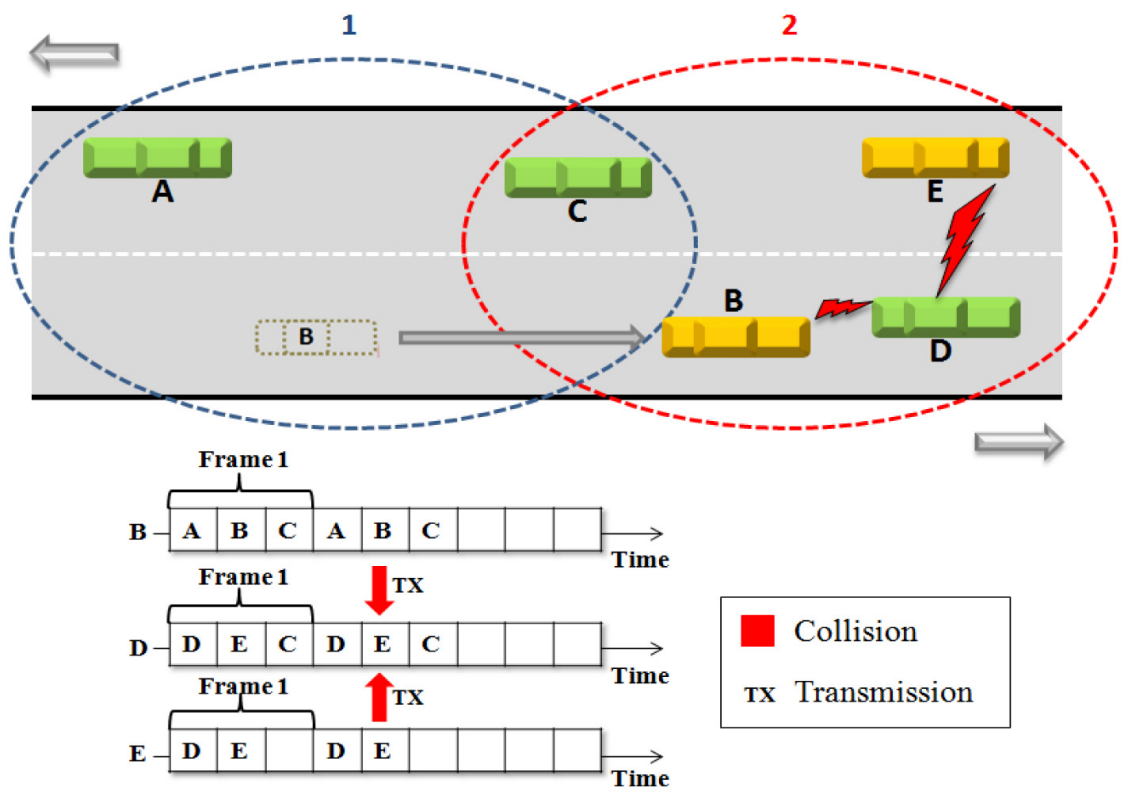

Figure 2 Merging collision problem

The One-Hop neighbouring Collision (OH-Collision) occurs when the same time slot is used by two neighbouring vehicles belonging to neighbouring clusters. Figure 3 shows an example of $\mathrm{OH}$-collision situation, when vehicle $\mathrm{C}$ in cluster 1 and vehicle D in cluster 2 are using the same time slot. Since $\mathrm{C}$ and $\mathrm{D}$ are within transmission range of each other, then a collision will occur at vehicle $\mathrm{C}$ and $\mathrm{D}$.



Figure 3 One-Hop neighbouring Collision ( $\mathrm{OH}-\mathrm{Collision})$ 


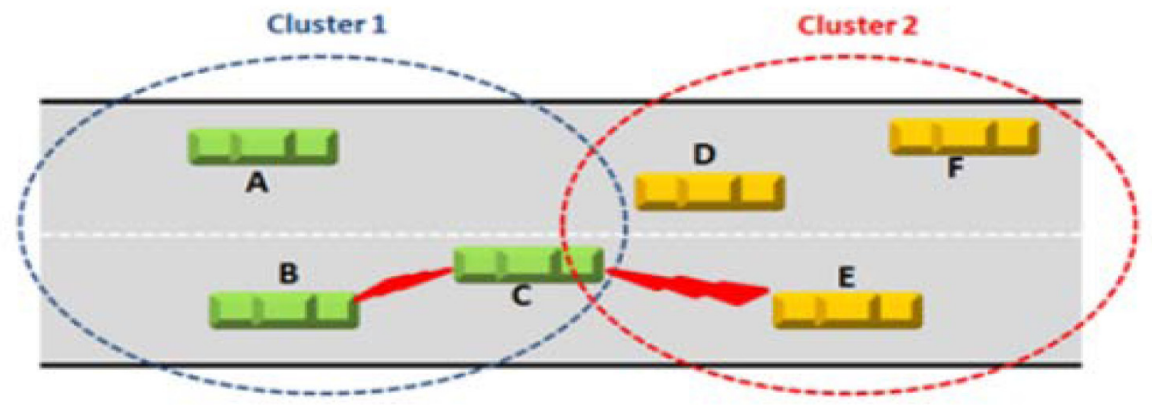

Figure 4 Hidden Node Collision (HN-Collision)

On the other hand, the Hidden Node Collision (HN-Collision) occurs when two vehicles are in range to communicate with another node, but not within transmission range of each other. Let us consider a situation in Figure 4 when vehicle B in cluster 1 and vehicle $\mathrm{E}$ in cluster 2 are using the same time slot. Since these two vehicles are outside transmission range of each other, a collision will occur at vehicle $\mathrm{C}$ in the cluster 1 .

\section{ASAS Protocol Description}

ASAS strategy is based on a time division multiple access (TDMA) method, in which the medium is divided into frames and each frame, is divided into time slots. Only one vehicle is allowed to transmit in each time slot. This proposed strategy is centralized in stable cluster heads that continuously adapt to a high dynamic topology. The main idea is to take the direction and position of the vehicles into consideration in order to decide which slot should be occupied by which vehicle. The allocation of time slots is based on the requests from the vehicles in their HELLO messages, which are used by the cluster head to calculate the slot transmission schedule. The strategy is robust in the sense that it provides an efficient time slot reservation without intra-cluster and intercluster interferences. In this section, we address two important challenges: Cluster formation and the TDMA slot assignment mechanism for intra-cluster and inter-cluster communications.

\subsection{Cluster Formation}

Clustering technique is the process that consists to divide all vehicles in a network into organized groups called clusters. Several algorithms have 
been proposed for cluster formation that take into account the specific characteristics of VANET network such as [11] and [16]. We have proposed a cluster formation algorithm based on information of the vehicle's position and direction, and which uses the Euclidean distance to divide the vehicles into clusters. To provide more stable clusters, our cluster formation scheme takes into account the direction of vehicles, i.e. only vehicles moving in the same direction can be members of the same cluster. If the direction is not taken into account in a highway environment with two ways, the vehicles that are moving in opposite direction to the cluster head will only be part of the cluster for a very short time and a new cluster will have to be formed almost immediately. Through the Euclidean distance and transmission range (i.e. the DSRC range is $1 \mathrm{~km}$ ), we can decide whether two vehicles can be grouped in the same cluster.

Initially, all vehicles are in the Undecided State US. To divide the network into clusters, each vehicle broadcasts its state (direction, position and speed) to notify its presence to its one-hop neighbours. Then, based on the received messages each vehicle can build its one-hop neighbouring list. To determine the most stable $\mathrm{CH}$, the elected cluster head is a vehicle which has the minimum average distance, the closet speed to the average speed and the maximum number of neighbouring vehicles. All the vehicles that are within transmission range of the elected $\mathrm{CH}$ become $\mathrm{CMs}$ and not allowed to participate in another cluster head election procedure. Once the clusters heads are elected, they maintain two sets of vehicles (see Figure 5): F (Front) and B (Back).

- $\mathrm{B}$ is a set of vehicles that are behind of the $\mathrm{CH}$

- F is a set of vehicles that are ahead of the $\mathrm{CH}$

Let $C_{i}$ a cluster of size $m$ and of cluster head $C H_{i}$ defined by position $(x, y, z)$.

- $F_{i}=\left\{V_{1 \leq i \leq m}\left(x^{\prime}, y^{\prime}, z^{\prime}\right), x^{\prime} \geq x\right\}$

- $B_{i}=\left\{V_{1 \leq i \leq m}\left(x^{\prime}, y^{\prime}, z^{\prime}\right), x^{\prime}<x\right\}=C_{i}-F_{i}$

After the cluster heads are elected as shown in Figure 5, each cluster head manages a local TDMA MAC frame. Moreover, after a cluster member CM receives its slot allocation from its cluster head, it transmits safety or control messages only during this slot and receives safety messages during the other time slots. 




\subsection{TDMA Slot Assignment in ASAS}

In the scope of this work, we assume that each vehicle is equipped with a positioning system e.g. GPS (Global Positioning System) which can provide an accurate real-time three-dimensional position (latitude, longitude and altitude), direction, velocity and exact time. The synchronization among vehicles may be performed by using GPS timing information.

\subsubsection{System Architecture}

A vehicle is said to be moving in a left (right) direction if it is currently heading to any direction from north/south to west (east), as shown in Figure 6. Based on this definition, if two vehicles are moving in opposite directions on a two-way road, it is certain that one vehicle is moving in a left direction while the other vehicle is moving in a right one [13]. In ASAS, the channel access time is partitioned into frames and each frame is divided into ABS period and CRP period. The ABS period consists of a set of time slots where each time 




Figure 6 System Architecture

slot can be used by one vehicle only ( $\mathrm{CM}, \mathrm{CG}$ or $\mathrm{CH})$ to broadcast a safety messages or other control messages such as topology management. The ABS period uses TDMA method as its channel access scheme. As defined in [13], to avoid merging collision problem, each ABS frame is divided into two sets of time slots, the first set is used by vehicles moving in left direction (see Figure 6) and the other is used by vehicles moving in right direction. The CRP period uses the contention-based method CSMA/CSA as its channel access scheme. During the CRP, if a vehicle needs to broadcast a message, it sends a request to the cluster head $\mathrm{CH}$ to reserve a periodic time slot. Then the $\mathrm{CH}$ responds to each reservation request from vehicle and assigns an available time slot in each ABS frame. We assume that each set of time slots Right or Left is partitioned into three subsets of time slots: $L, R$ and $N$, as shown in Figure 6.

- $\mathrm{L}$ is the subset of time slots reserved for vehicles belong to the F set of vehicles.

- $\mathrm{R}$ is the subset of time slots reserved for vehicles belong to the B set of vehicles.

- $\mathrm{N}$ is the subset of unused time slots, in which all vehicles in the cluster remain inactive.

Moreover, to avoid the inter-cluster interference problem, the orders of the time slots subsets are different between neighbouring clusters. Hence, ASAS 
can reduce the inter- cluster and intra-cluster interferences without the use of other complex techniques such as CDMA, FDMA, SDMA and OFDMA.

\subsubsection{TDMA slot reservation}

In this section we provide a detailed description of our TDMA slot allocation strategy. When a vehicle $\mathrm{V}$ needs to access network, it first sends a reservation request to the cluster head $\mathrm{CH}$ for a periodic time slot. When $\mathrm{CH}$ receives the reservation request and depending on the vehicle position, it determines whether the current time slot belongs to the L, R set, and then it selects to $\mathrm{V}$ the first available slot as its owner slot. Each cluster head $\mathrm{CH}$ determines its distribution of three subsets of time slots "MAP" according to the MAPs of their neighbouring clusters. The $\mathrm{CH}$ can obtain the MAP information of the neighbouring clusters heads through the cluster gateways $\mathrm{CG}$. Once a $\mathrm{CH}$ has selected a time slot for a $\mathrm{CM}$, it sends a reservation which includes the slot identifier. However, ASAS requires that every $\mathrm{CH}$ should periodically send frame information FI to its two neighbouring clusters heads via its CGs. This information contains the following (see Figure 7):

1. CH-ID indicates the identifier of $\mathrm{CH}$ that sends the FI packet.

2. $\operatorname{MAP}\{\{\mathrm{R}, \mathrm{L}, \mathrm{N}\} ;\{\mathrm{L}, \mathrm{N}, \mathrm{R}\}$ or $\{\mathrm{N}, \mathrm{R}, \mathrm{L}\}\}$.

3. The sizes of $\mathrm{R}, \mathrm{L}, \mathrm{N}$ subsets.

4. The state of each time slot reserved for its moving direction.

The second information element is transmitted only once time and the third is transmitted if the cluster head updates the size of the $L, R$ or $N$ subsets. Unlike other slots reservation techniques based on FI broadcasts where each vehicle must determine the set of time slots used by all vehicles within its two-hop neighbourhood in order to acquire a time slot. In our reservation technique, the $\mathrm{CH}$ discovers the available slots while requiring less overhead than the others techniques. Moreover, the $\mathrm{CH}$ knows also all the time slots which likely to cause a collision at the transmission channel (i.e. $\mathrm{N}$ set). As shown in Figure 8, especially in the frame information of cluster head number 6 (FI-6), there is one available time slot for new vehicle moving behind of the cluster head. However, the reservation of any time slot which identifier belong to

\begin{tabular}{|c|c|c|c|c|c|c|}
\hline CH-ID & \multicolumn{2}{|c|}{ MAP } & Size & $\begin{array}{c}\text { Slot Status } \\
\text { SS_[1] }\end{array}$ & $\ldots . .$. & $\begin{array}{c}\text { Slot Status } \\
\text { SS_[K/2] }\end{array}$ \\
\hline
\end{tabular}

Figure 7 Frame information 


\begin{tabular}{|c|c|c|c|c|c|c|c|c|c|c|c|c|c|c|c|c|c|c|}
\hline $\begin{array}{c}\text { Time Slot } \\
\text { ID } \\
\begin{array}{c}\text { Cluster } \\
\text { ID }\end{array}\end{array}$ & $\mathbf{1}$ & $\mathbf{2}$ & $\mathbf{3}$ & $\mathbf{4}$ & $\mathbf{5}$ & $\mathbf{6}$ & $\mathbf{7}$ & $\mathbf{8}$ & $\mathbf{9}$ & $\mathbf{1 0}$ & $\mathbf{1 1}$ & $\mathbf{1 2}$ & $\mathbf{1 3}$ & $\mathbf{1 4}$ & $\mathbf{1 5}$ & $\mathbf{1 6}$ & $\mathbf{1 7}$ & $\mathbf{1 8}$ \\
\hline $\mathbf{1}$ & & & & & & & & & & 1 & 2 & 3 & 4 & 5 & & $\mathrm{~N}$ & $\mathrm{~N}$ & $\mathrm{~N}$ \\
\hline $\mathbf{2}$ & & & & & & & & & & 9 & 10 & & $\mathrm{~N}$ & $\mathrm{~N}$ & $\mathrm{~N}$ & 6 & 7 & 8 \\
\hline $\mathbf{3}$ & 18 & & & $\mathrm{~N}$ & $\mathrm{~N}$ & $\mathrm{~N}$ & 15 & 16 & 17 & & & & & & & & & \\
\hline 4 & 11 & 12 & 13 & 14 & & & $\mathrm{~N}$ & $\mathrm{~N}$ & $\mathrm{~N}$ & & & & & & & & & \\
\hline
\end{tabular}

Figure 8 An example of slot assignment

[13 . . 15] may cause a collision. When all the slots in L or R subsets are busy, the $\mathrm{CH}$ must communicate with its two neighbouring clusters heads to reserve a time slot in the $\mathrm{N}$ set for new vehicles respectively belonging to the F or B set.

The time slots are allocated according to the vehicle's movement and positions. By using a centralized approach we change the slot allocation process from random reservations to optimal allocations, which can improve the convergence performance of the MAC protocol and achieves an efficient broadcast service for a successful delivery of real-time safety information.

\subsubsection{Release of TDMA Slot}

If a cluster head does not receive a beacon message after a specific time from a $\mathrm{CM}$ to signal its presence, then the $\mathrm{CH}$ immediately releases the time slot allocated to that $\mathrm{CM}$ and it removes this $\mathrm{CM}$ from its cluster members list (i.e. the F or B set).

\section{Performance Evaluation}

In this section, we evaluate the effect of transmission range variation and we carry out a comparison of ASAS with the DMMAC and the VeMAC protocols. The simulation is based on an event-driven simulator implemented using Java language. We have used VanetMobiSim [8] to generate a mobility scenario.

\subsection{Mobility Scenarios and Simulation Parameters}

The mobility scenarios implemented for the highway are with two-way and different density levels (see Figure 5). The vehicles are moving at different speeds and have different transmission ranges. During simulation 
Table 1 System parameters for simulation

\begin{tabular}{ll}
\hline Highway length & $2 \mathrm{~km}$ \\
Ways & 2 \\
Lanes/way & 2 \\
Transmission range/scenario & $\{150,350,550,750,1000\} \mathrm{m}$ \\
Slots/ Frame & 50 \\
Slots for left direction & 25 \\
Slots for left direction & 25 \\
Slot duration & $1 \mathrm{~ms}$ \\
Simulation time & $120 \mathrm{~s}$ \\
Number of vehicles/scenario & 60 \\
Speed mean value & $100 \mathrm{~km} / \mathrm{h}$ \\
Speed standard deviation & $30 \mathrm{~km} / \mathrm{h}$ \\
\hline
\end{tabular}

System parameters for simulation

time, each vehicle moves at a constant speed, and the number of vehicles on the highway remains constant. Table 1 summarizes the simulation parameters.

\subsection{Performance Metrics and Simulation Results}

We have evaluated ASAS based on the following performance metrics:

- MR-Collision rate: MR-Collision rate is defined as the average number of merging collisions.

- AC-Collision rate: The AC-Collision rate is computed as the average number of access collisions.

- IC-Collision rate: The IC-Collision rate is defined as the average number of inter-cluster collisions due to $\mathrm{HN}-\mathrm{Collision}$ and $\mathrm{OH}-\mathrm{Collision}$. However for the DMMAC and the VeMAC protocols, the IC-collision rate is defined as the rate of collisions between the adjacent sets of two-hop neighboring vehicles that are moving in the same direction.

Due to the high dynamic topology, the number of clusters varies during the simulation time (new cluster are added and clusters are merged) and this variation should be as low as possible. Thus the cluster formation algorithm proposed reduces the number of new clusters created due to the high mobility of the vehicles. Moreover, it creates stable clusters and keeps the current clusters as stable as possible.

Figure 9, shows the rate of access collisions as function of different vehicle densities. Notice that there is no access collisions generated by ASAS, whereas both the DMMAC and the VeMAC protocols suffer from collisions. This can 




Figure 9 The access collision rate as a function of vehicle density

be explained by the centralized nature of the ASAS protocol, where cluster heads are in charge of time slots allocation. The DMMAC protocol as well as the VeMAC protocol generate higher rate of access collisions than ASAS, particularly for high traffic load. These results show the effectiveness of the ASAS technique.

Figure 10, shows the rate of merging collision for ASAS, VeMAC and DMMAC as function of transmission range. The ASAS protocol eliminates all merging collisions for the different transmission ranges studied. This comes from the fact that ASAS protocol assigns different sets of time slots to vehicles moving in opposite directions. The figure shows also that the merging collision rate is reduced by $100 \%$ compared to the DMMAC and VeMAC protocols.

In Figure 11 we depict the rate of IC-Collisions for the ASAS, VeMAC and DMMAC protocols. It is easy to see that ASAS have a lower rate of IC-Collisions than the two other protocols. The IC-Collision rate is reduced by $50 \%$ compared to VeMAC and by $5-15 \%$ compared to DMMAC. The main reason is that ASAS protocol allocates distinct sets of time slots to vehicles moving ahead and behind the cluster head. Consequently, ASAS protocol reduces collisions between neighboring clusters, which decreases the rate of Inter-cluster collisions compared to the DMMAC, and VeMAC protocols. We can also see that the IC-Collision rate decreases as the transmission range increases. This is because increasing the transmission range, decreases the number of clusters in the network and thus the inter-cluster collision rate will automatically decrease. We can conclude that ASAS protocol performs successfully under the DSRC architecture since the transmission range in DSRC is equal to $1000 \mathrm{~m}$. However, in case of low transmission range (less 




Figure 10 The merging collision rate as function of transmission range



Figure 11 The inter-cluster collision rate as function of transmission range

than $250 \mathrm{~m}$ ) the DMMAC protocol presents better results than ASAS, because in this case, the large number of clusters increases the rate of inter-cluster collisions. 


\section{Conclusion and Future Work}

This paper has studied the MAC protocol challenges to satisfy the requirements of real time and reliable broadcast of safety messages while achieving the fairness of the channel access. We have designed the Adaptive TDMA Slot Assignment Strategy (ASAS) to overcome these problems in which the assignment of time slots to vehicles is performed by the cluster heads in order to avoid any access collision problems. From the simulation results we conclude that this strategy achieves an efficient reservation and utilization of the available time slots without access collisions and decreases the rate of merging collisions as well as the rate of the inter-cluster collisions caused by the hidden node problem. Compared with the DMMAC and VeMAC protocols, ASAS generates a lower rate of transmission collisions in different transmission ranges and traffic load conditions. ASAS achieves this without having to use expensive spectrum management mechanisms such as CDMA or FDMA.

In future work, we will study the effect of various speeds and vehicles densities on the performance of ASAS. In addition, the dynamic adjustment of the length of the three subsets will be scrutinized. Moreover, we plan to extend ASAS to support multichannel operation and to provide reliable broadcast on both the control and service channels. In addition, we will carry out extensive simulations comparison with the IEEE 802.11p standard that operates with the DSRC architecture.

\section{References}

[1] K. Hafeez, L. Zhao and Z. Niu, Distributed Multichannel and MobilityAware Cluster-Based MAC Protocol for Vehicular Ad Hoc Networks, IEEE Trans. On Vehicular Technology, vol. 62, no. 8, oct 2013.

[2] Federal Communications Commission, FCC 99-305, FCC Report and Order, October 1999.

[3] The FCC DSRC (Dedicated Short Range Communications) web site. http://wireless.fcc.gov/services/its/dsrc/.

[4] T. Wu and S. Biswas, Reducing Inter-cluster TDMA Interference by Adaptive MAC Allocation in Sensor Networks, Sixth IEEE International Symposium on a World of Wireless Mobile and Multimedia Networks (WoW-MoM'05), 2005.

[5] CAR 2 CAR Communication Consortium. http://www.car-to-car.org/. 
[6] 802.11p-2010-IEEE standard for information technology-Telecommuni cations and information exchange between systems-local and metropolitan area networks - specific requirements part 11 : Wireless LAN medium access control (MAC) and physical layer (PHY) and physical layer (PHY) specifications amendment 6 : Wireless access in vehicular environments. 2010.

[7] 802.11-2007 - The institute of electrical and electronics engineers IEEE standard for information technology - telecommunications and information exchange between systems - local and metropoli-tan area networks specific requirements. Part 11: Wireless LAN medium access control (MAC) and physical layer (PHY) specifications.

[8] VanetMobiSim project, home page (2010). http://vanet.eurecom.fr (accessed: May 29, 2010).

[9] Q. Tse, Improving Message Reception in VANETs, in Proc. of International Conference on Mobile Systems, Applications and Services (MobiSys), June 2009.

[10] TGp. http://www.ieee802.org/11/Reports/tgp update.htm.

[11] P. Fan, P. Sistla and P. C. Nelson, Theoretical analysis of a directional stability-based clustering algorithm for vanets, Vehicular Ad Hoc Networks, 2008.

[12] F. Borgonovo, L. Campelli, M. Cesana and L. Fratta, Impact of user mobility on the broadcast service efficiency of the ADHOC MAC protocol, Proc. IEEE VTC, vol. 4, pp. 2310-2314, 2005.

[13] H. A. Omar, W. Zhuang and L. Li, VeMAC: A TDMA-Based MAC Protocol for Reliable Broadcast in VANETs, IEEE TRANSACTIONS ON MOBILE COMPUTING, VOL. 12, NO. 9, September 2013.

[14] Y. Günter, B. Wiegel, and H. Grossmann, Cluster-based medium access scheme for vanets, Intelligent Transportation Systems Conference, 2007. ITSC 2007. IEEE, pp. 343-348, Oct 2007.

[15] M.S. Almalag, S. Olariu and M.C. Weigle, TDMA cluster-based MAC for VANETs (TC-MAC), Proc. IEEE WoWMoM, pp. 1-6, 2012.

[16] C. Shea, B. Hassanabadi and S. Valaee, Mobility-based clustering in VANETs using affinity propagation, in IEEE Globecom, 2009.

[17] F. Liu N. Lu, Y. Ji and X. Wang, DMMAC : A dedicated multi-channel MAC protocol design for VANET with adaptive broadcasting, in Wireless Communications and Networking Conference (WCNC), 1-6, Sydney, Australia, 2010. 


\section{Biographies}

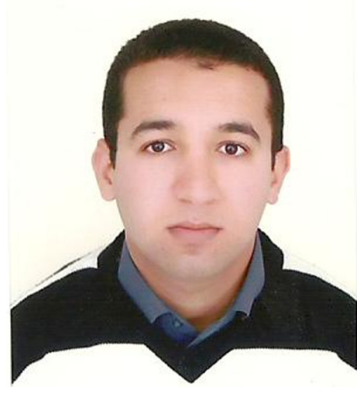

Mohamed HADDED received Bachelor's Degree in Computer Science from the Faculty of Science of Gabes and MS degree in Computer Sciences and Information Systems from the Higher Institute of Computer Science and Mathematics. He is currently a PhD student at the National School of Computer Science of Manouba. His research interests include vehicular communications, mobility management, and Quality of Service.

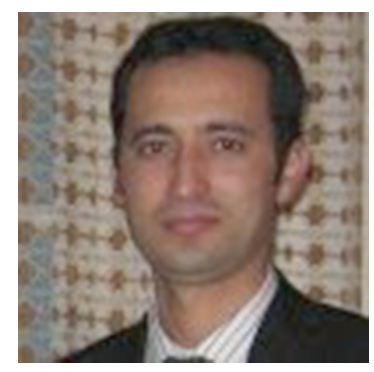

Dr. Rachid Zagrouba is an assistant professor from September 2008 at university of Tunis El Manar (Tunisia). He received his Computer Science Engineering and Master degrees from the National School of Computer Science (ENSI) in 2001 and 2002, respectively. At the end of 2003, he joined the RSM research team at Telecom Bretagne in Rennes (France) to prepare a Ph.D. in Computer Science which he has defended on December 2007. During 2006 and 2007, he was an ATER (Attach Temporaire d'Enseignement et de Recherche) at the University of Rennes 1. From September 2003 to September 2006, he was with the Mobile Communications team of orange Lab in Rennes as a research engineer. He was involved in several French-funded and IST FP6/7 projects. He served as a technical reviewer of several international 
conferences and journals. He is the Ph.D. Supervisor of three Ph.D. students in the area of computer networking, wireless and cellular networks, vehicular communications, and mobility management.

Dr. Anis Laouiti received his $\mathrm{PhD}$ in computer science from the Versailles University, France, in 2002. He had been doing his research during and after his Phd at INRIA/Hipercom team, before he joined the TELECOM Sud-Paris, France, as an associate professor in 2006. His research interests include unicast/multicast routing protocols for MANET, and vehicle to vehicle communications. He was involved in the IETF-MANET working group and he is one of the co-authors of the OLSR routing protocol.

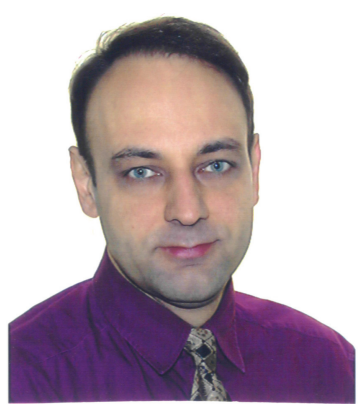

Pr. Paul Muhlethaler was born in february 1961. He graduated from Ecole Polytechnique in 1984. He received his PhD in 1989 from Paris Dauphine university and its habilitation in 1998. He is researcher at INRIA since 1988. $\mathrm{He}$ is now research director at INRIA where he co-founded the HiPERCOM team with Philippe Jacquet. His research topics are mainly around protocols for networks with a speciality in wireless networks. He had also a few, often referenced, results on scheduling issues. In wireless networks, he has actively worked at ETSI and IETF for the HiPERLAN and OLSR standards. He is now following the European standardization for vehicular networks. Another important aspect of his activity concerns models and performance evaluations. He was the first to carry out optimizations of CSMA protocols in Multihop Ad Hoc Networks. With F. Baccelli and B. Blaszczyszyn he derived a complete model of an Aloha multihop ad hoc net-work. Interestingly this model lead to the design of one of the first multihop ad hoc network offering a throughput scaling according the Guta and Kumar's famous law. He is now particularly interested in deeply understanding the achievable performance of multihop 


\section{M. Hadded et al.}

ad hoc networks and in tracking all the possible optimization ways of such networks. In 2004, he received the pres-tigious price "Science et Dfense" for his work on Mobile Ad Hoc Networks. He was among the first contributors for the OLSR protocol in 1997.



Pr. Leila Azouz Saïdane is Professor at the National School of Computer Science (ENSI), at The University of Manouba, Tunisia and the Chair person of the PhD Commission at ENSI. She was the Director of this school and the supervisor of the Masters Degree program in Networks and Multimedia Systems. She is the head of RAMSIS group of CRISTAL Research Laboratory (Center of Research in Network and System Architecture, Multimedia and Image Processing) at ENSI. She collaborated on several international projects. She is author and co-author of several papers in refereed journals, magazines and international conferences. 